\title{
A Robust Methodology for Prediction of Trust and Reputation Values
}

\author{
Farookh Khadeer Hussain \\ Digital Ecosystems and Business \\ Intelligence Institute \\ Curtin University of Technology \\ Perth, WA, Australia \\ 0061-8-92669268 \\ F.Hussain@curtin.edu.au
}

\author{
Elizabeth Chang \\ Digital Ecosystems and Business \\ Intelligence Institute \\ Curtin University of Technology \\ Perth, WA, Australia \\ 0061-8-92669272 \\ E.Chang@curtin.edu.au
}

\author{
Omar Hussain \\ Digital Ecosystems and Business \\ Intelligence Institute \\ Curtin University of Technology \\ Perth, WA, Australia \\ 0061-8-92669275 \\ O.Hussain@curtin.edu.au
}

\begin{abstract}
In this paper, we present the $\mathrm{FC}$ direct trust value-based decision making methodology, for making direct trust value based decisions regarding interactions in (a) a given context and during the current time slot, and (b) a given context and at a future time slot. The direct trust value-based decision making methodology models the context specific nature of trust and the dynamic nature of trust to make direct trust value-based decisions regarding interactions. Additionally in this paper, we present the FC reputation-based trust decision making methodology, for making reputation-based trust decisions regarding interactions, if direct trust value-based decisions cannot be made. The FC reputationbased trust decision making methodology can make reputationbased trust decisions regarding interactions in (a) a given context and during the current time slot, and (b) a given context and at a future time slot.
\end{abstract}

\section{Categories and Subject Descriptors}

E.m [MISCELLANEOUS]: Trust, I.2 [Distributed Artificial Intelligence]: Intelligent agents, Multiagent systems, K.1 [THECOMPUTER INDUSTRY]: Suppliers.

\section{General Terms}

Algorithms, Measurement, Performance, Design, Reliability, and Experimentation.

\section{Keywords}

Trust, Reputation, Prediction, Dynamic Nature of Trust, Dynamic Nature of Reputation, Markov Model, Dynamic Models

\section{INTRODUCTION}

It is possible that a given entity has access to previous ' $n$ ' reputation values for another entity corresponding to ' $n$ ' consecutive time slots, as shown below in Figure 1 . In this case, it would be useful to utilize the previous reputation values to predic future reputation value of the trusted entity, since the reputation

Permission to make digital or hard copies of all or part of this work for personal or classroom use is granted without fee provided that copies are not made or distributed for profit or commercial advantage and that copies bear this notice and the full citation on the first page. To copy otherwise, or republish, to post on servers or to redistribute to lists, requires prior specific permission and/or a fee

SWS 08, October 31, 2008, Fairfax Virginia USA

Copyright 2008 ACM 978-1-60558-292-4/08/10 $\$ 5.00$ values may change over time. The reasons that the reputation values vary over time slots are as follows:

1. The behaviour of the trusted entity in question varies over the time slots.

2. Corresponding to the change in the behaviour of the trusted entity, the trustworthiness values assigned to it by other agents who have interacted with it in different time slots have varied.

3. Since the reputation value of the entity in a given time slot, is the combination of the trustworthiness values assigned by the third party recommendation agents to the trusted entity who have interacted with it in that time slot, corresponding to the change in the behaviour of the entity over different time slots, its reputation value too has varied over the time slots.

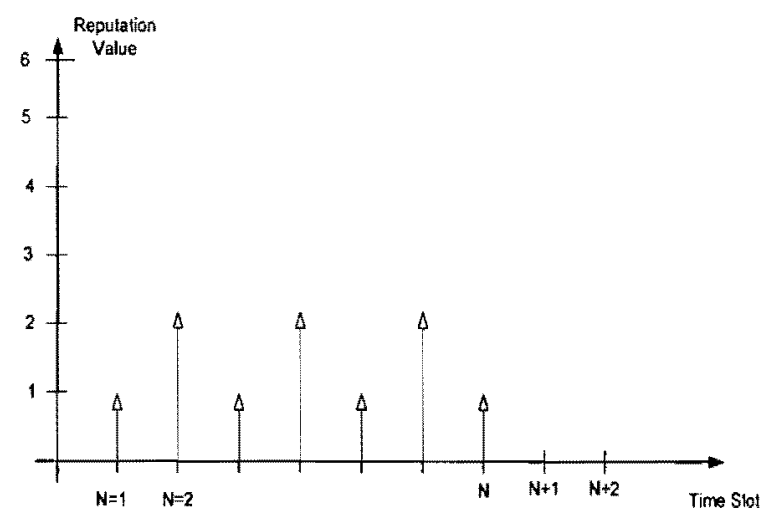

Figure 1. Reputation values of an entity over a sequence of time slots

The change in the behaviour of the trusted entity could be due to several sources, as listed below [1]:

(a) The service provider may provide different quality of service depending on the load being experienced at a particular time. A similar argument could also be applied to the product manufacturer

(b) There could be a change of management or a change of policy in the provision of the service by the service provider, resulting in a change in the quality of the provided service. A 
similar argument could also be applied to the product manufacturer

(c) It is possible that the quality of service provided by a given service provider slowly deteriorates over time. A similar argument could be applied to the quality of product engineered by a product manufacturer.

It is possible that the service provider is engaging in cyclical fraud by periodically providing services of lower quality. A similar argument could be applied to the product manufacturer. In this paper, we explain the working of our proposed prediction method by explaining the process of predicting the future reputation value of an entity. It is, however, important to note here that the prediction method can be used to determine the future trustworthiness value of an entity as well in order to make direct trust value based decisions regarding interactions. The process of predicting the future reputation value of an entity is more complex than the process of determining the future trustworthiness value of an entity and hence, in this paper, we explain process of predicting the future reputation value of an entity.

In the existing literature there are various approaches that mode trust and reputation from various perspectives [2-21]. However, the major shortcoming of these proposed methods in the literature is that a significant majority of them treat both trust and reputation as static variables. However as is clearly evident from the above discussion, trust and reputation are dynamic variables and $w$ need to mathematically model them as such. In particular the existing literature does not propose any method for predicting the future trust values or reputation values, based on the past values. In Section 2, we present an overview of the FC direct trust valuebased decision making methodology. This is a comprehensive methodology that can be used for context-based trust decision making both at a current time slot and at a future time slot. In Section 3, we present an overview of the FC reputation-based trust decision making methodology. Additionally, from Section 4 Section 7, we present and discuss the mathematical working of these methodologies in detail. In Section 8, we describe the simulation set up that we have used for validation purposes. In Section 9 , the results are presented. Finally, Section 10 concludes the paper.

\section{FC DIRECT TRUST VALUE-BASED} DECISION MAKING METHODOLOGY

In this section, we propose the FC direct trust value-based decision making methodology, by which the trusting agent can make direct trust value-based decision of interactions with a given trusted entity, by taking into account both the dynamic nature of trust and the context specific nature of trust. The proposed FC direct trust value-based decision making methodology can

1. Make direct trust value-based decisions regarding interactions in a given context and during the current time slot, (and)

2. Make direct trust value-based decisions regarding interactions in a given context and during a future time slo by predicting the context specific and time slot specific trustworthiness value of an entity.

\subsection{FC Direct Trust Value-based Decision Making Methodology in a given context and current time slot}

If the trusting agent intends to make a trust-based decision of interaction with a trusted entity, in a given context and during the current time slot, then the first step is to determine whether it has knowledge about the context specific and current time slot specific trustworthiness value of the trusted entity. If that is the case, then the trusting agent can make a direct trust value-based decision regarding interaction based on the context specific and current time slot specific trustworthiness value of the trusted entity

In case the trusting agent does not have knowledge about the context specific and current time slot specific trustworthiness value of the trusted entity, it then makes use of the trustworthiness values of the trusted entity in time slots prior to the current time slot and in the given context, to determine the trustworthines value during the current time slot. Assuming that the trusting agent has knowledge about the trustworthiness values of the trusted entity in the given context, and in at least half the time slots in the time space, it can make use of the prediction methods proposed in this paper, to determine the current trustworthiness value of the trusted entity and subsequently make a direct trust value-based decision of interaction, based on the trustworthiness value.

However, if that is not the case, then the trusting agent needs to determine the context specific and current time slot specific reputation value of the trusted entity in order to make a reputation-based trust decision regarding interaction. In Section 3.1, we propose the $\mathrm{FC}$ reputation-based trust modelling methodology for making context specific and current time slo specific reputation-based trust decisions regarding interactions.

\subsection{FC Direct Trust Value-based Decision Making Methodology in a given context and future time slot}

If the trusting agent intends to make a trust-based decision regarding interaction with the trusted entity, in a given contex and at a future time slot, then the first step it needs to do is to determine if it has knowledge about the context specific trustworthiness values of the trusted entity in at least half the time slots with in the time space. If that is the case then the trusting agent makes use of the prediction methods proposed in this pape to predict the future trustworthiness value of the trusted entity and subsequently make a direct trust value-based decision regarding interaction based on the predicted trustworthiness value

In case then the trusting agent does not have knowledge of the context specific trustworthiness values of the trusted entity in at least half of the time slots with in the time space, then it needs to determine the reputation value(s) of the trusted entity in those time slots (time slots in which it does not have knowledge about direct trust value) and subsequently it makes use of the prediction methods, to make a trust-based decision regarding interaction. 


\section{FC REPUTATION-BASED TRUST DECISION MAKING METHODOLOGY}

In this section, we introduce the FC reputation-based trust decision making methodology, which is a mathematical means of modelling both the dynamic nature of reputation and contex specific nature of reputation when making a reputation-based trus decision of interaction. The proposed FC reputation-based trust decision making methodology can:

1) Determine the reputation value of an entity in a given context and during the current time slot, in order to make a reputation-based trust decision regarding interaction, if a direct trust value-based decision of interaction in the given context and during the current time slot cannot be made (and)

2) Predict the reputation value of an entity in a given contex and at a future time slot, in order to make a reputation-based trust decision regarding interaction, if a direct trust valuebased decision of interaction in the given context and the future time slot cannot be made.

The proposed method of predicting the future reputation value of an entity is a modified version of the Markov model which is widely used to make weather predictions, power load predictions etc. The motivation for predicting the future reputation value of an entity based on the Markov model is as follows [1]:

1) The Markov model is a mathematical model and hence has its roots in a mathematical theory rather than being an ad-hoc formulation, like the decay based approach

2) The Markov model can be seamlessly used to model the changes in the reputation values or trustworthiness values of an entity over time.

3) The Markov model has been a well tested and widely applied theory about making predictions, in various fields

In the next section, we discuss in detail our proposed FC reputation-based trust modelling methodology by which a given trusting agent can make a reputation-based trust decision regarding interaction with a given trusted entity in a given contex and during the current time slot. In Section 32 , we discuss our proposed methodology by which a given trusting agent can make a reputation-based trust decision regarding interaction with given trusted entity in a given context and at a time slot in the future.

In the case where the trusting agent does not have knowledge of the context specific trustworthiness values of the trusted entity in any of the time slots within the time space, then it needs to determine the reputation values of the trusted entity in all the time slots in the time space and subsequently it makes use of the prediction method (proposed in Section 3.2), to make a reputation trust-based decision regarding interaction.

\subsection{FC Reputation-based Trust Decision Making Methodology in a given context and at the current time slot}

In our proposed FC reputation-based trust decision making methodology, if a given trusting agent intends to make a reputation-based trust decision of interaction with a given trusted entity, in a given context and at the current time slot, then the firs step it needs to take is to issue a reputation query for the trusted entity in question. Once a reputation query has been issued, the rusting agent, takes up the role of the reputation querying agent and the trusted entity takes up the role of the reputation queried entity. We propose that the reputation query in this case should contain the following:

1) the identity of the reputation queried entity; (and)

2) the context in which the reputation querying agent intends to make the reputation-based trust decision regarding interaction with the reputation queried entity; (and)

3) the current time slot during which the reputation querying agent intends to make the reputation-based trust decision regarding interaction with the reputation queried entity. The current time slot is specified by specifying the time spot from which it starts and the time spot when it ends

The third party recommendation agent(s), on receipt of the reputation query, communicate recommendation(s) about the reputation queried entity. The recommendation(s) communicated by the third part recommendation agent(s), would correspond to the trustworthiness value(s) assigned by the third party recommendation agent(s) to the reputation queried entity in the context and time slot specified in the reputation query. The replies from the third party recommendation agents are additionally qualified by the time spot of their interaction.

The reputation querying agent collects the recommendation(s) from different third party recommendation agent(s). It then weed out those recommendation(s) which do not fall within the specified time slot (if any). It then uses the witness trustworthiness value of the third party recommendation agent(s) to weed out recommendation(s) from the untrustworthy known agent(s). It then combines the recommendation(s) obtained from trustworthy known agent(s) and the unknown agent(s) using the following Equation I to determine the reputation queried entities reputation value at the current time slot.

Reputation $(m, A)=\left(\sum_{i=1}^{K} W T V(i) \circ(\right.$ Recommendation $\left./ m, i, A, t]\right) / K$

$+\beta^{*}\left(\sum_{j=1}^{i} \operatorname{Re} c o m m e n d a t i o n s[m, j, A, t]\right) / L$

....Equation 1

Where,

- $\quad K$ and $L$ are the number of trustworthy known agents and unknown agents, respectively, in current time slot (say $N$ );

- $A$, denotes the context in which the reputation querying agen is determining the reputation value of the reputation queried entity;

- $m$, denotes the reputation queried entity:

- Reputation ( $m, A)$, is the computed reputation value of the reputation queried entity ' $m$ ' in context ' $A$ '. The domain of the computed reputation value is between reputation level 1 and reputation level 6 ;

- Recommendation $[m, i, A, t]$, is the recommendation from the third party recommendation agent ' $i$ ', about the reputation queried entity ' $m$ ', when they interacted with each other a time spot ' $\mathrm{t}$ ' and in context ' $\mathrm{A}$ '; 
- $W T V(i)$, represents the witness trustworthiness value of third party recommendation agent ' $i$ ', as perceived by the reputation querying agent.

- $\theta$, is an operator that adjusts the recommendation communicated by a given third part recommendation agen with its witness trustworthiness value. We henceforth cal this operator as the Recommendation Adjustment Operator. The algebra for this operator would be discussed is not described in this paper due to space constraints

$\beta$, is a weighting factor, which gives an appropriate weighting to the recommendations communicated by the unknown agents. We have fixed $\beta$ to 0.2 . The first term in Equation 1, adjusts all the recommendation(s) from the trustworthy known agent(s) by the witness inustworthiness value of the third party recommendation agent communicating the recommendation and subsequently aggregates all the adjusted recommendation(s) from the trustworthy known agents. The second term aggregates the recommendations from the unknown agents. Since the reputation querying agent does not have any previous experience of soliciting recommendation from the unknown agents, it cannol discard their recommendation as untrustworthy recommendations. Neither can it assign the same weight to the recommendations communicated by unknown agent as it does to the recommendations communicated by trustworthy known agents. The factor $\beta$ is used to weight the aggregated value of the recommendations from the unknown agents appropriately.

It may be the case that the reputation querying agent is unable to determine the reputation value of the reputation queried entity during the current time slot because:

- It obtained no recommendations about the reputation queried entity from the third party recommendation agents. As a result the computed repute value is 0 .

- It obtained recommendations about the reputation queried entity from the third party recommendation agents but all of these were from untrustworthy known agents. As a result, the computed repute value is 0 .

In order to address this scenario, the reputation querying agent needs to determine the reputation value of the reputation queried entity, in all the time slots excluding the current time slot and excluding the time slots for which it has knowledge about the direct trust value of the reputation queried entity. Subsequently, it makes use of the prediction method (proposed in the next section), to make a trust-based decision of interaction.

\subsection{FC Reputation-based Trust Decision Making Methodology in a given context and at a future time slot}

In this section, we present the working of our prediction method, for predicting the future reputation value of a given entity. We explain the working of our proposed prediction method for predicting the future reputation value of an entity, because the process involved in predicting the future reputation value of an entity is more complex than the process of predicting the future trustworthiness value of an entity. Predicting the future reputation value of an entity involves determining its reputation values in the previous time slots and then making use of the prediction method. On the contrary, predicting the future trustworthiness value of an entity involves just making use of the prediction method proposed in this section.
The steps involved in the prediction of the future reputation value of an entity are as follows

1. Issue a reputation query about the trusted entity. The format of the reputation query issued to make a reputation-based trust decision regarding interaction in a future time slot, is explained in Section 3.3.

2. Construct the reputation series. This is explained in Section 3.4.

3. Determine if the reputation series is a 'stationary reputation series' or a 'non-stationary reputation series'. The definition of the terms 'stationary reputation series' and 'non-stationary reputation series' are explained in Section 3.5 along with the method of classifying a given reputation series into one of these two types.

4. If the reputation series is a stationary reputation series, then predict the future reputation value by making use of a modified version of the Markov model. Subsequently make reputation-based trust decision regarding interaction based on the predicted reputation value. This is explained in Section 4.

5. If the reputation series is non-stationary, then it may either be seasonal or trend or noisy. In case of a non-stationary reputation series, the reputation querying agent first needs to determine if the reputation series is a seasonal reputation series. We formally define the term 'seasonal reputation series' in Section 3.5. In this paper, for simplicity purposes we assume that a reputation series can be either seasonal or can have trend in it but not both. However, the method can be extended to the case where a reputation series has both seasonal components and varying trends. If a reputation series cannot be classified as a seasonal reputation series or trend reputation series, then we treat that reputation series as a noisy reputation series. In the case of seasonal reputation series, the reputation querying agent makes a prediction of the future reputation value of the reputation series, while accounting for the seasonality present in the reputation series. This is explained in Section 5.

6. If the reputation series is neither stationary nor seasonal, then the reputation querying agent needs to determine whether it is a trend reputation series. We formally define the term "trend reputation series" in Section 3.6. If the reputation series is a trend reputation series, then the reputation querying agent needs to carry out trend component analysis and make a prediction about the reputation value in a future time slot accounting for the trend. This is explained in Section 6.

7. If the reputation series is non-stationary and does not have seasonal variations and trend, then the reputation series is random reputation series. We formally define the term 'random reputation series' in Section 7. In this case, the reputation querying agent needs to make a prediction about the reputation value in a future time slot accounting for the noise in the reputation series. This is explained in Section 7.

If a given trusting agent is predicting the future trustworthiness value of an entity, then it needs to carry out steps $3-7$.

\subsection{Issue a reputation query about the trusted} entity

The first step in the $\mathrm{FC}$ reputation-based trust decision making methodology for predicting the reputation value in a given context 
at a future time slot is for the trusting agent to issue a reputation query about the trusted entity. The contents of the reputation query in this case are, however, different from the one specified in the last section. Apart from the identity of the reputation queried entity and the context in which the reputation querying agent intends make a reputation-based trust decision regarding interaction, the reputation query should in this case contain the time space based on which the reputation querying agent intends to determine the future reputation value of the reputation queried entity. The time space represents the whole duration of time ove which the reputation querying agent wishes to track the reputation value of the reputation queried entity in order to determine its future reputation value. The time space is specified in the reputation query by specifying the time spot from which it starts and the time spot when it ends.

The third party recommendation agent(s) on receipt of the reputation query communicate recommendation(s) about the reputation queried entity, qualified by the time spot of their interaction with the reputation queried entity.

\subsection{Construct the reputation series}

The second step in the FC reputation-based trust decision making methodology for predicting the reputation value in a given context and at a future time slot is to construct the reputation series. We define reputation series as a sequence of reputation values of given entity in a given context corresponding to a sequence of time slots [1].

In order to construct the reputation series, the reputation querying agent, on receipt of the recommendation(s) from the third party recommendation agent(s) classifies them into different time slots according to the time spot of the interaction, specified in the recommendation. From the recommendations classified into each time slot, the reputation querying agent then weeds out the recommendations from untrustworthy known agent(s) and subsequently aggregates the recommendations from the trustworthy known agent(s) and unknown agent(s) in each time slot according to the following formulae:

Reputation $(m, A, N)=\left(\sum_{i=1}^{k} W T V(i) \circlearrowright(\right.$ Recommendation $\left.[m, i, A, t])\right) / K$

$+\beta^{*}\left(\sum_{j=1}^{L} \operatorname{Re}\right.$ commendations $\left.[m, j, A, t]\right) / L$

.....Equation 2

Equation 2 is similar to Equation 1 with the only exception being the parameter ' $N$ ' on the left hand side of the equation, which is used to denote a given time slot.

As a result of applying the above process, the reputation querying agent would have a sequence of reputation values of the reputation queried entity in a given context and corresponding to a sequence of time slots. Mathematically, the reputation series belonging to an entity $A$ over ' $M$ ' consecutive time slots can be represented as follows:

$$
R_{p}^{A} \quad p=1, \ldots M
$$

....Equation 3
Once the reputation querying agent has determined the reputation series of the reputation queried entity, for objective and accurate prediction of the future reputation value, it determines whether the reputation series can be classified as a stationary reputation series or a non-stationary reputation series.

\subsection{Classify the reputation series as stationary or non-stationary}

We define a stationary reputation series as a reputation series in which all the reputation values are exactly same. We define a nonstationary reputation series as a reputation series in which at leas one reputation value differs from the other reputation values in the reputation series

The objective of carrying out the above analysis is to determine whether or not the reputation queried entity has been maintaining a given level of reputation, consistently over time. If the reputation series of a reputation queried entity is stationary, then the future reputation value can be predicted by taking into accoun the stationary property of the reputation series. In the next section, we propose a method to classify a reputation series as stationary reputation series or non-stationary reputation series

The reputation querying agent needs to carry out the following steps in order to classify a given reputation series as being either a stationary reputation series or non-stationary reputation series:

1. Fit a line over the whole reputation series using linear regression method.

2. Compute the gradient of the fitted line.

3. If the gradient of the line fitted over the reputation series is 0 then the reputation series can be classified as a stationary reputation series. In case of a stationary reputation series, the reputation querying agent can directly predict the future reputation value, using the method proposed in Section 4 .

4. However, if the gradient of the line fitted over the reputation series is not equal to 0 , then the reputation series can be classified as a non-stationary reputation series and further analysis needs to be carried out to determine if the variations of the reputation values in the reputation series can be classified as being seasonal or trend or noise and predict the future reputation value accounting for the particular variation.

\subsection{Classify the non-stationary reputation} series as seasonal or trend or noisy

If the reputation values in the reputation series are varying over time, we classify it as a non-stationary reputation series. The variations could take many forms, outlined below, each of which would require different methods to determine the specific variation and predict the future reputation value while accounting for the specific variation.

The variations could indicate a recurring regular pattern also termed as seasonality variation in the reputation series [I]. If this is the case, then the future reputation value should be predicted while accounting for the seasonality variation. We define seasonal variation as the occurrence of either a peak reputation value(s) or a low reputation value(s) or both, recurting at regular intervals of time within a reputation series. We define a peak reputation value in a reputation series as an increase in the reputation value relative to the immediately previous reputation 
value (prior to the increase) possibly maintaining the increase in reputation value for multiple time slot(s) and then returning back to the reputation value prior to the occurrence of the increase. We define a low reputation value as a decrease in the reputation value relative to the immediately previous reputation value possibly maintaining the decrease in reputation value for multiple time slot(s) and then returning to the reputation value prior to the occurrence of the decrease. It should be noted that a given reputation series, exhibiting seasonal variations, could have just peak reputation values(s) or low reputation values(s) or both. We define a seasonal reputation series as a reputation series that has seasonal variations in it. The assumptions that we make about the seasonality variations should be noted

1. The peak (and/or) the low reputation value(s) should occur at regular intervals of time within a given reputation series, in order for it to qualify as seasonal variations.

2. The peak (and/or) the low reputation value(s) occurring within a given reputation series at regular intervals of time should be of the same value.

In Section 5, we propose an algorithm to determine seasonality variations in the reputation series if they exist and predict a future reputation value, while accounting for the seasonality variations in the reputation series.

In contrast, the variations could indicate a regular downward or upward movement of reputation values also termed as trend in the reputation series. We define trend variations as either a decrease or an increase in the reputation values in a reputation series over sequence of time slots by some amount. It should be noted that in contrast to seasonality variations, where the reputation values eithe increase or decrease by a specific amount and then return to the reputation value previous to the increase or decrease, in trend, the reputation values either keep on increasing by some amount or keep on decreasing by some amount. If the amount of decrease or the amount of increase from one time slot to the next time slot is the same over the whole reputation series, then we could fit the reputation series by a linear line with goodness of fit equal to one. For simplicity purposes, we will make this assumption here, but there is no difficulty in using the polynomial curve in its place. We define a trend reputation series as one that has trend variations in it.

If this is the case, then the future reputation value should be predicted while accounting for the trend. In Section 6, we propose an algorithm to determine whether the trend in the reputation series exists, and predict a future reputation value, while accounting for the trend in the reputation series.

If the variations cannot be explained or classified as being seasonal variations or trend variations, then the reputation series is random or noisy. We define a noisy reputation series as a sequence of reputation values without any regular recurring pattern or trend. In this case, the future reputation value should be predicted while accounting for the noise in the reputation series. We propose an algorithm to determine the noise in the reputation series and predict a future reputation value, while accounting for the noise, in Section 7

\section{PREDICTING FUTURE REPUTATION VALUE IF THE REPUTATION SERIES IS STATIONARY}

If the reputation series is stationary, then the process of determining the future reputation value, and making a reputationbased trust decision based on the predicted reputation value, is straightforward and will be based on a discrete time Markov process which is in the steady state. The steps involved in predicting the future reputation value when the reputation series is stationary are as follows [1]

1. Construct the transition matrix from the reputation series.

2. Construct the current state vector from the reputation series.

3. Determine the future state vector of the reputation series from the current state vector and the transition matrix

4. Determine the reputation value at the future time slot using the future state vector and make a reputation-based trust decision of interaction based on the predicted reputation value.

In this section, we explain each of these steps in sequential order.

\subsection{Constructing the Transition Matrix - T} from the reputation series

We define a transition matrix as a means to capture and denote the probability of the reputation of an entity transiting from one reputation level to another on the basis of its past behavior [1]. It should be noted here that the past behavior of a given entity is captured using the reputation series. To determine the probability of an entity transiting from reputation level 1 to reputation level 2 we find the ratio between the number of times that the entity has transited from reputation level $\mid$ to reputation level 2 and the total number of times it has transited from reputation level 1 to any other reputation level in the reputation series. Mathematically, the probability of the reputation level of an entity transiting from level $i$ to level $j$ is shown in Equation 4 below:

$$
p_{i j}\left(t_{N}\right)=\frac{n_{i j}}{n_{i}}
$$

Equation 4

Where, $n_{i j}$ is the total number of transitions from $i^{\text {th }}$ reputation level to the $j^{\text {th }}$ reputation level and

$$
n_{i}=\sum_{j} n_{i j}
$$

Equation 5

It should be noted that $\sum_{j} p_{i j}=1$. The transition matrix $\mathrm{T}$, would be a $6 \times 6$ matrix with rows of the matrix corresponding to the reputation level at time slot ' $\mathrm{P}$ ' and columns corresponding to the reputation level at time slot ' $\mathrm{P}+1$ ' of a given entity. An element in the matrix denotes the probability of that entity transiting from the reputation level corresponding to the row in which the elemen occurs, to the reputation level corresponding to the column in which the element occurs, over a sequence of consecutive time slots. The number of rows and columns in the transition matrix are fixed at 6 , each corresponding to the six different possible levels of reputation as discussed in Chang et al. [1]. 
Mathematically the elements of the transition matrix are represented as follows:

$$
p_{i j}\left(t_{N}\right) \quad i, j=1, \ldots, 6
$$

Equation 6

Where, 6 is the number of reputation levels, as explained earlier

\subsection{Constructing Current State Vector-C from the reputation series}

In order to denote or model or represent the reputation value of the reputation queried entity at the current time slot (denoted as ' $N$ '), we propose the concept of a current state vector. We define the current state vector of a reputation queried entity as the vector that models and represents the reputation value of the reputation queried entity at the current time slot.

The current state vector shows the reputation queried entities reputation value at the time slot $N$. It will be a $l \times 6$ matrix, since there are six different possible reputation levels that a given reputation queried entity could be at during the current time slot. Mathematically, the current state vector is represented as follows:

$$
C=\left[C_{1}, C_{2}, C_{3}, C_{4}, C_{5}, C_{6}\right]
$$

Equation 7

We represent a given reputation queried entity's current state vector using Equation 7, denoting it with a 1 corresponding to the reputation value of an entity at the time slot $\mathrm{N}$ and all the other reputation levels are denoted with 0

\subsection{Constructing the Future State Vector- F}

Once the reputation querying agent has determined the transition matrix and the current state vector for the reputation queried entity, it is now in a position to determine the future probable reputation value of the reputation queried entity at time slot ' $\mathrm{N}+\mathrm{l}$ '. In order to do so, the reputation querying agent multiples the transition matrix (T) of the reputation queried entity with its current state vector $(C)$ to determine its future state vector $(F)$

The computed future state vector denotes the probabilities of the reputation queried entity behaving with a reputation level $i$ at time slot ' $\mathrm{N}+\mathrm{l}$ '. Mathematically, we denote the future state vector of a given reputation queried entity as follows:

$$
F=\left[f_{1}, f_{2}, f_{3} \ldots \ldots, f_{6}\right]
$$

Equation 8

Where,

$F$ is the future state vector; (and)

$f_{1}, f_{2}, f_{3}, f_{4}, f_{3}$ and $f_{6}$ denote the probability of the trusted entity having a trustworthiness value or reputation value of ' 1 ', ' 2 ', ' 3 ', ' 4 ', '5' and ' 6 ' respectively.

Mathematically, the future state vector $\mathrm{F}$ can be obtained with:

$$
\mathrm{F}=\mathrm{C} \times \mathrm{T} \text {. }
$$

Equation 9
From the future state vector, the reputation queried entity's future reputation value (at time slot ' $\mathrm{N}+\mathrm{l}$ ') is determined as that reputation level to which the reputation queried entity has the highest probability of transiting.

In our proposed reputation-based trust decision making methodology, the reputation querying agent decides to go ahead and interact with the reputation queried entity only if the reputation queried entity's computed future reputation level is $\geq 5$ A threshold reputation level of 5 is chosen because, a reputation level 5 and reputation level 6 correspond to 'good reputation' and 'very good reputation respectively', which means that the trusted entity can be interacted with. Reputation levels $1,2,3$ and 4 however, suggest otherwise. Once the reputation-based trus decision of interaction has been made and the reputation querying agent has chosen an entity for interaction, the reputation querying agent then takes up the role of the trusting agent and the chosen reputation queried entity takes up the role of the trusted entity.

\section{PREDICTING FUTURE REPUTATION VALUE IF THE REPUTATION SERIES HAS SEASONALITY VARIATIONS}

In our proposed methodology, if the gradient of the linear line fitted over the reputation series is not equal to zero (which means that the reputation series is a non-stationary reputation series) then the reputation querying agent first needs to carry out a test to determine whether the reputation series has seasonal variations in

We propose an algorithm that is basically a pattern recognition algorithm over the reputation series to determine whether it has any seasonal variations. It should be noted here that when we refer to the term seasonal variations it is under the definition and assumptions made previously.

The algorithm that we propose in this section, would firstly determine whether a given reputation series contains seasonal variation or seasonal cycle and if that is the case, it then predict the future reputation value of the reputation series while accounting for the seasonal variations. However, if there are no seasonal variations in the reputation series and the gradient of the line fitted over the reputation series is not equal to zero, the reputation series may either contain trend or it may be a noisy reputation series. In that case, in our proposed methodology, the reputation querying agent subsequently carries out a test to determine whether the reputation series exhibits trend. The test is proposed and outlined in the next section.

The proposed algorithm for determining whether a given reputation series can be classified as a seasonal reputation serie works as follows:

1. It tries to find out whether there is any seasonal variation (or seasonal cycle) in the reputation series.

2. If seasonal variation has been found to exist in the reputation series, it then tries to determine where or in which part of the seasonal variation, the current reputation value at time slot 'N' fits.

3. Once the reputation value at time slot ' $N$ ' has been fitted in the seasonal variation, the future reputation value at time slot ' $\mathrm{N}+\mathrm{l}$ ' is determined by following the (previous) seasonal variation. 
The working of our proposed algorithm for determining seasonal variation in the reputation series (if any) and predicting the future reputation value while accounting for the seasonal variation is as follows:

1. It takes the last reputation value in the reputation series (the reputation value at time slot ' $N$ '). Let us call this value the 'test series', for further discussion purposes.

2. Should the test series (reputation value at time slot $\mathrm{N}$ during the first stage of the algorithm) be a seasonal variation by itself, then all the reputation levels to which the reputation queried entity has transited to from the test series would be exactly the same. If this is the case, then the future predicted reputation value is the reputation level to which the reputation queried entity has always transited whenever it was at the reputation level specified by the test series.

3. If step 2 above is not satisfied, then determine if reputation values at time slots $\mathrm{N}-1$ and $\mathrm{N}$ of the reputation series form a seasonal variation. In this case the reputation values at time slots $\mathrm{N}-1$ and $\mathrm{N}$ form the test series. Let us assume for discussion purpose that the concatenated reputation values at time slots $\mathrm{N}-1$ and $\mathrm{N}$ is denoted as $\mathrm{R}-1 \| \mathrm{R}$. Should $\mathrm{R}-1 \| \mathrm{R}$ (the test series) form a seasonal variation then, the reputation value to which the reputation queried entity has transited in the past from $R-1 \| R$ would always be the same. If this is the case, then the future reputation value at time slot ' $N+l^{\prime}$ is specified by the reputation level to which the reputation queried entity has transited whenever it was in a state of R-1 |R.

4. If step 3 is not satisfied, then iteratively repeat the process as described above each time with the test series in each iteration being the concatenation of the test series in the previous iteration and the reputation value corresponding to the immediately previous time slot to the one already existing in the test series. It should be noted that this reputation value should be appended as the most significant bit in the test series. Carry the process iteratively till either a seasonal variation is found or the above check for seasonality variations has been carried out over all the reputation values from time slots from $1 \ldots N$. In the former case, there is a seasonal variation in the reputation series and we make use of the seasonal variation which has occurred in the past, determine at which point the reputation value at the current time slot falls, and extrapolate along the seasonal cycle or seasonal variation. In the latter case, there is no seasonal variation in the reputation series and the reputation querying agent needs to determine whether the reputation series can be classified as a trend reputation series.

In our proposed FC reputation-based trust decision making methodology, if the reputation series exhibits seasonality variations, then the reputation querying agent decides to go ahead and interact with the reputation queried entity only if the reputation queried entities predicted future reputation level, accounting for the seasonality variations is $\geq 5$. Once the future reputation value of the reputation queried entity has been determined and the reputation querying agent has chosen an entity for interaction, the reputation querying agent then takes up the role of the trusting agent and the chosen reputation queried entity takes up the role of the trusted entity.

\section{PREDICTING FUTURE REPUTATION VALUE IF THE REPUTATION SERIES HAS TREND IN IT}

In our proposed methodology, if the gradient or slope of the linear line fitted over the reputation series is not equal to zero and the reputation series cannot be classified as a seasonal reputation series, the next step is to determine if it can be classified as a trend reputation series. In case the reputation series cannot be classified as a trend reputation series, then the given reputation series is a random reputation series or noisy reputation series.

In our proposed method, in order to determine whether a given reputation series has trend in it, the reputation querying agent computes the 'goodness of fit' value of the linear line fitted over the reputation series. If the non-stationary reputation series canno be classified as a seasonal reputation series and if the 'goodness of fit' value for the linear line over the reputation series is 1 , then the reputation series exhibits trend. The trend in the reputation series could indicate an upward increase in the reputation values, denoting that the $\mathrm{OOS}$ provided by the service provider or the QoP of the product has been increasing as a function of time slots. Alternatively, the trend could indicate a downward decline in the reputation values, denoting that the QoS provided by the service provider or the $\mathrm{QoP}$ of the product has been decreasing as a function of time slots. The linear fitted line over the reputation series would manifest the upward or downward trend in the reputation series. Our objective it to extrapolate the trend (whether it is upward or downward) and predict the future reputation value while accounting for the trend.

Mathematically, the linear fitted reputation series is represented as follows:

$$
r=f(n)
$$

..Equation 10

Where,

' $r$ is the reputation value,

' $n$ ' is used to denote a time slot,

$\mathrm{f}(\mathrm{n})$ is the linear function that has been fitted over the reputation series as a function of time slots.

The simplest way to predict the future reputation value of the reputation queried entity while accounting for the trend is to determine the value of the function $f(n)$ at time slot ' $N+1$ '. Mathematically this is represented as follows:

$$
\mathrm{R}=\mathrm{f}(\mathrm{N}+\mathrm{I})
$$

Equation 11

Where $R$ is the reputation value of the reputation queried entity at time slot ' $\mathrm{N}+1$ '.

\section{It should be noted that if}

1. the computed value for $R$ using the above equation is $<1$, then $R$ takes a value of 1 ;

2. the computed value for $R$ using the above equation is $>6$, then $R$ takes a value of 6 ;

3. the computed value for $R$ using the above equation is rounded off to the nearest integer value in the domain of $[1-6]$

In our proposed FC reputation-based trust decision makine methodology, if the reputation series exhibits trend, then the reputation querying agent decides to go ahead and interact with the reputation queried entity only if the reputation queried entities 
computed future reputation level, accounting for the trend (the value of $R$ from Equation 11) is $>5$. Once the future reputation value of the reputation queried entity has been determined and the reputation querying agent has chosen an entity for interaction, the reputation querying agent then takes up the role of the trusting agent and the chosen reputation queried entity takes up the role of the trusted entity.

\section{PREDICTING FUTURE REPUTATION VALUE IN CASE OF RANDOM REPUTATION SERIES}

If a non-stationary reputation series cannot be classified as being either seasonal reputation series or trend reputation series, it is a serjes of random reputation values. We regard such a series as a noisy reputation series or random reputation series. Our proposed methodology in the case of a noisy reputation series or random reputation series determines the noise present in the reputation series and predicts the future reputation value accounting for the noise. The steps involved in our proposed methodology for predicting a future reputation value while accounting for the noise in a noisy reputation series is as follows:

1. Determine the mean of the random reputation series and subtract the mean from each reputation value in the reputation series. Let us assume that the reputation value in the reputation series at time slot ' $t$ ' is denoted as $R(t)$ Furthermore, let us assume that the value at time slot't' in the reputation series after subtracting the mean $(M)$ from it is denoted as $S(t)$. $S(t)$ is determined as shown below in Equation 12

$$
S(t)=R(t)-M \quad \text { for all } t
$$

Equation 12

The value $S(t)$, can give us a measure of the noise represent in the reputation value at time slot ' $\mathrm{t}$ '.

2. Extrapolate the linear line fitted over the reputation series to the next time slot. Let us assume that the extrapolated reputation yalue be denoted as $\mathrm{R}^{\prime}$.

3. Augment or adjust the value $R^{\prime}$ by an amount equal to $S(N)$ (i.e. noise in the reputation series at time slot $N$ )

Mathematically, the predicted reputation value at time slot ' $\mathrm{N}+\mathrm{l}$ ', if the reputation series is random is determined using Equation 13:

$$
R(N+1)=R^{\prime} \Delta S(N)
$$

Equation 13

Where,

$\Delta$ is the reputation scaling operator. The algebra for this operator would be discussed is not described in this paper due to space constraints.

The logic behind the above steps in predicting the future reputation value of a noisy reputation series is as follows

1. The linear fitted line over the reputation series would correspond to the mean of the reputation series.

2. Extrapolate the linear line over the reputation series (denoting its mean) over to the next time slot.
3. Augment the extrapolated reputation value (from step 2, above) by an amount equal to noise present in the last reputation value of the reputation series

The reputation querying agent may wish to take into account the average value of the noise present in the previous ' $n$ ' time slots in the reputation series, leading to Equation 14 , below.

$$
R(N+1)=R^{\prime} \Delta((S(N)+S(N-1)+\ldots . . S(N-n)) / n)
$$

Equation 14

It is possible to weight the individual $S(t)$ values differently as well, by introducing weights in the above equation. In this thesis we make use of Equation 13 to determine the future reputation value at time slot ' $\mathrm{N}+\mathrm{I}^{\mathrm{X}}$ if the reputation series is noisy.

In our proposed FC reputation-based trust decision making methodology, if the reputation series is random, then the reputation querying agent decides to go ahead and interact with the reputation queried entity only if the reputation queried entities computed future reputation value, computed according to Equation 13 is $\geq 5$. Once the reputation querying agent has chosen an entity for interaction, the reputation querying agent then takes up the role of the trusting agent and the chosen reputation queried entity takes up the role of the trusted entity.

\section{SIMULATION SETUP}

In this section, we present the various phases involved in the TSN Single Agent System, along with the results obtained from the TSN Single Agent System. The various phases in the TSN Single Agent System are described in the following section.

\subsection{Initialization Phase in TSN Single Agent System}

In the initialization phase, the user inputs a reputation series, with which it intends to predict the future reputation value. The reputation series can be thought of as the exogenous parameters for the TSN single agent system.

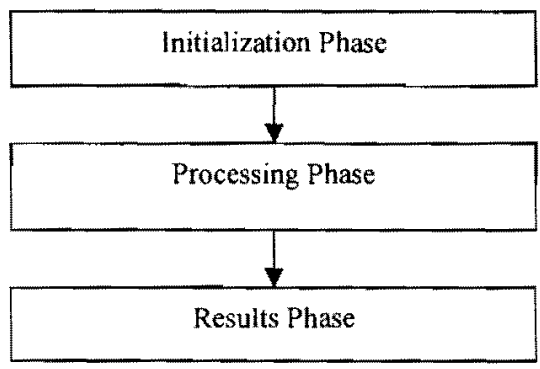

Figure 2. Flow Chart of the TSN Single Agent System

\subsection{Processing Phase in TSN Single Agent} System

The TSN Single Agent System makes use of the approach explained and outlined previously, to detemine whether the reputation series exhibits seasonality. If the reputation series exhibits seasonality, it determines the future reputation value of the reputation series while accounting for the seasonality presen in the reputation series. Secondly, if the reputation series does not 
exhibit seasonality, then the TSN single agent system makes use of the approach explained and outlined previously to determine whether the reputation series exhibits trend. If this is the case, then the future reputation value is predicted while accounting for the trend present in the reputation series. Finally, if the reputation series does not exhibit both trend and seasonality, then the reputation series exhibits noise. In this case, the TSN single agent system makes use of the approach explained and previously to determine and quantify the amount of noise present in the reputation series and predict a future reputation value while accounting for the noise

\subsection{Results Phase in TSN Single Agent System}

The aim of this phase in the TSN single agent simulation is to present graphically the predicted future reputation values of the reputation series by making use of the $\mathrm{FC}$ reputation-based trust modeling methodology. We then discuss whether the predicted future reputation by the TSN single agent simulation takes into account the trend, seasonality or noise present in the reputation series.

\section{RESULTS}

We first input a reputation series that contains seasonality, as shown in Figure 3 below. The reputation series that we have input is 123456123456 . As we can see, this reputation series is seasonal and the seasonal cycle present in the reputation series is

123456 . Given the seasonal cycle in the reputation series, the next reputation value of the reputation series, taking into accoun the seasonality cycle present in the reputation series, should be 1 . In order for the TSN single agent system to predict the next reputation value of the reputation series we press the "Go" button as shown below in Figure 3.

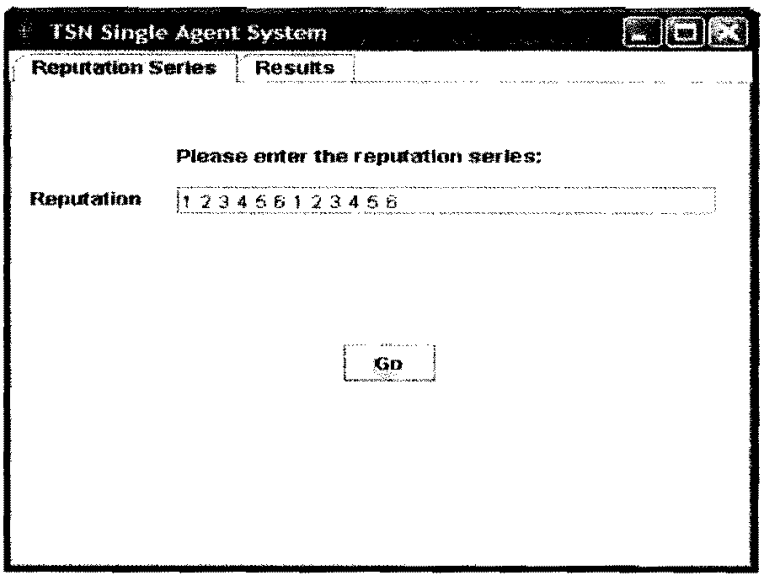

Figure 3. Inputting seasonal reputation series 123456123 456 into the TSN Single Agent System

Figure 4 shown the future predicted reputation value when the input reputation series is 123456123456 . As can be seen from the figure below, the TSN single agent system takes the seasonal cycle present in the reputation series into account and predicts the future reputation value as 1 .

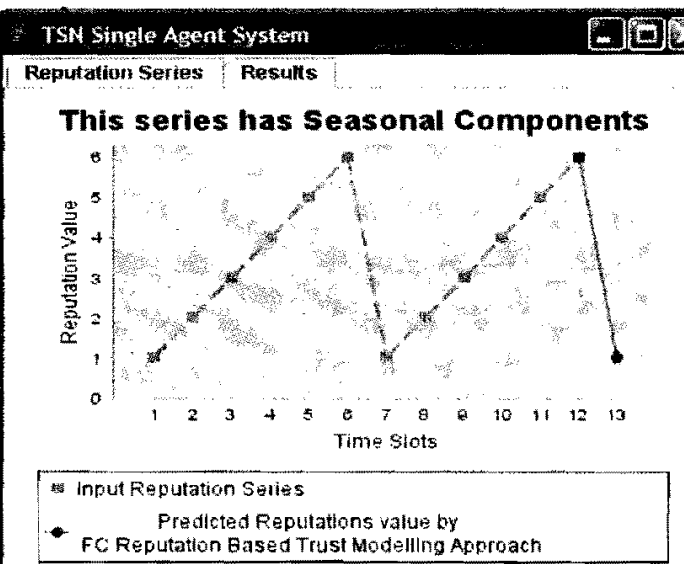

Figure 4. Predicted reputation value by the TSN single agent system when input is 123456123456

In order to further validate the accuracy of the predicted reputation value by the $\mathrm{FC}$ reputation-based trust modeling methodology when the reputation series has seasonality in it, we enter the reputation series as 5555556555555 (as shown in Figure 5)

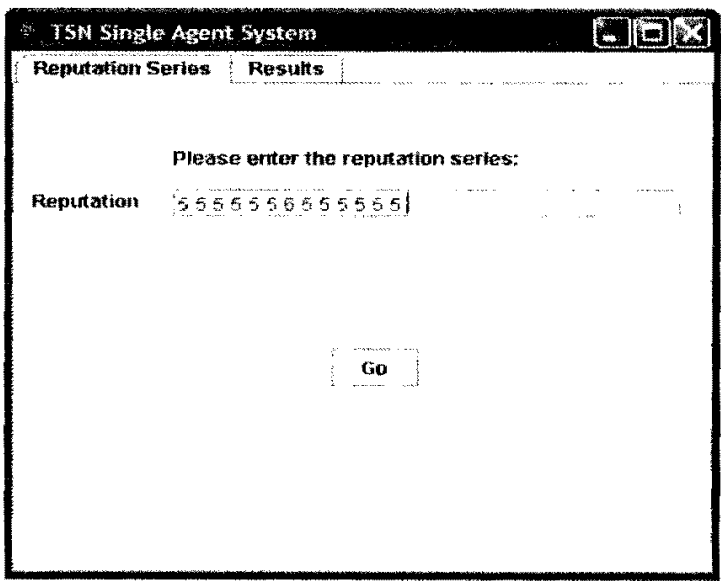

Figure 5. Inputting Seasonal Reputation Series 55555565 55555 into the TSN Single Agent System

As can be clearly seen, the reputation series is seasonal with the seasonal cycle being ' 5555556 '. Taking into account the seasonal cycle present in the reputation series, and since the current reputation value is 5 and all the previous five reputation values relative to the current reputation value are 5 , the next reputation value of the reputation series should be 6 . Figure 6 shows the predicted reputation value by the TSN Single Agent System when the input reputation series is 555555655555 5 . As we can see the predicted reputation value by the TSN Single Agent System is 6, which is predicted taking into account the seasonality present in the reputation series. 


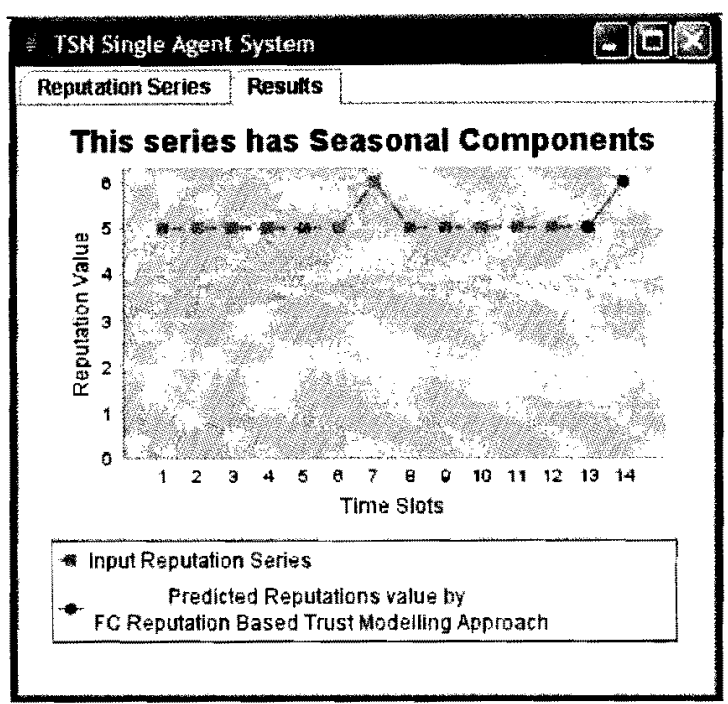

Figure 6. Predicted Reputation Value by the TSN Single Agent System when input is 5555556555555

It should be noted for the given reputation series, that from a reputation level of 5 the reputation series has transited to a reputation level of 5,10 times out of 12, with only once transiting to reputation level of 6 from a reputation level of 5 . However, the FC reputation-based trust modeling methodology is robust in detecting seasonal cycles present in the reputation series (if any) and predicting the future reputation value by extrapolating the seasonal cycle while overriding the Markov transitions where they are not applicable.

In order to validate the accuracy of the predicted reputation value by the FC reputation-based trust modeling methodology when the reputation series has trend in it, we enter the reputation series as 6 54 (as shown in Figure 7). As can be clearly seen, the reputation series has a downward trend. For this particulat reputation series, with a downward trend, it is more appropriate to extrapolate the downward trend rather than to make use of the reputation value in the current time slot. A downward extrapolation of the downward trend present in the reputation series to the next time slot would yield a reputation value of 3 . Figure 8 , shows the predicted reputation value when the input reputation series is 654 , by the TSN Single Agent System. As we can see the predicted reputation value by the TSN Single Agent System is 3, which is predicted taking into account the trend or rather the downward trend present in the reputation series.

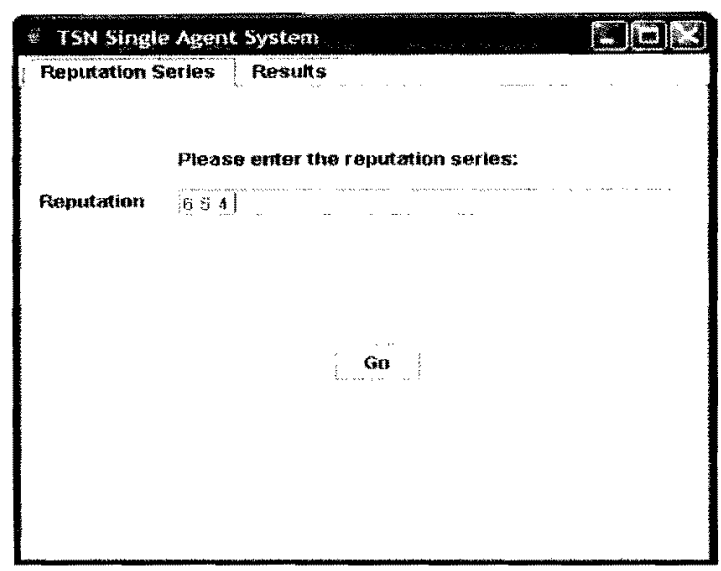

Figure 7. Inputting Trend Reputation Series 654 into the TSN Single Agent System

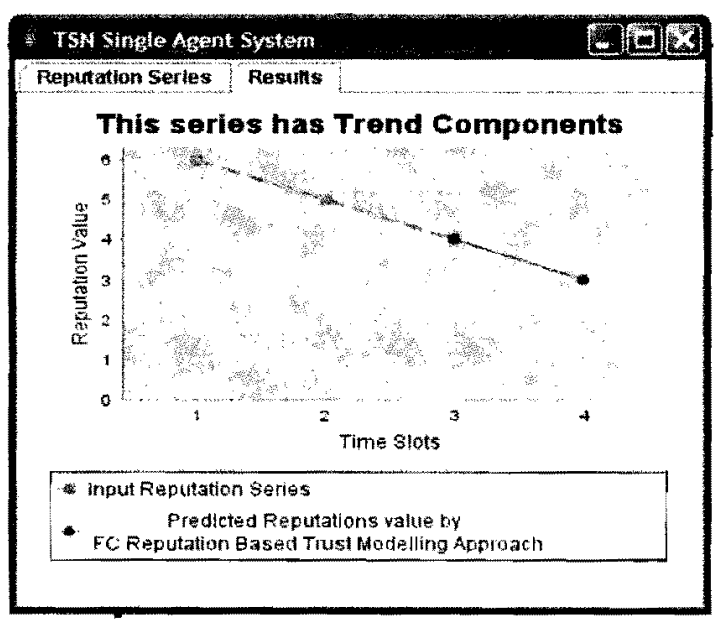

Figure 8. Predicted Reputation Value by the TSN Single Agent System when input is 654

\section{CONCLUSION}

In this paper we presented an overview of the FC direct trus value-based decision making methodology and $\mathrm{FC}$ reputation based trust decision making methodologies. They can be used for context-based trust decision making both at a current time slot and at a future time slot. The mathematical working of these methodologies was presented and explained in detail in this paper

Furthermore, the simulation set up that we have used for validation purposes was introduced and presented. Finally, the results obtained were presented and discussed.

\section{ACKNOWLEDGEMENTS}

The research work presented in this paper was supported by ARC Linkage Grant LP 0560346. Additionally, the authors would like to thank the support from Digital Ecosystems and Business Intelligence (DEBI) Institute 


\section{REFERENCES}

11] Chang, E. Dillon, T., and Hussain, F.K. 2005. Trust and Reputation for Service-Oriented Environments: Technologies for Building Business Intelligence and Consumer Confidence, Ed. John Wiley and Sons, U.K., ISBN: 0-470$01547-0,350$ pages.

[2] Damiani, E., Vimercati, S.D.C., Jajodia, S., Paraboschi, S. and Samarati, P. 2003. Balancing confidentiality and efficiency in untrusted relational DBMSs, Proceedings of the $10^{\text {th }} \mathrm{ACM}$ Conference on Computer and Communications Security (CCS 2003), Washingtion, USA, 27-30 October 2003, pp.93-102.

[3] Damiani, E., Vimercati, S.D.C., Finetti, M., Paraboschi, S, Samarati, P., and Jajodia, S. 2003. Implementation of a Storage Mechanism for Untrusted DBMSs, Proceedings of the $2^{\text {nd }}$ International IEEE Security in Storage Workshop (SISW 2003), Washingtion, USA, 31 October 2003 , pp.38-46

[4] Marsh, S. 1994 Formalising Trust as a Computational Concept, PhD thesis, Department of Mathematics and Computer Science, University of Stirling.

[5] Dellarocas, C. 2001. Analyzing the economic efficiency of eBay-like online reputation reporting mechanisms, Proceedings of the $3^{\text {rd }} \mathrm{ACM}$ Conference on Electronic Commerce (EC-2001), Tampa, USA, 14-17 October, 2001, pp. $171-179$

[6] Yu, B., Venkatraman, M., Singh, M.P. 1999. A Multiagent Referral System for Expertise Location, Proceedings of the AAAI Workshop on Intelligent Information Systems, Orlando, Florida, 18-22 July 1999, pp. 66-69.

[7] Abdul-Rahman, A., Hailes, S. 2000. Supporting Trust in Virtual Communities, Proceedings of the $33^{\text {rd }}$ Annual Hawai International Conference on System Sciences, Hawaii, USA, 4-7 January 2000, pp. 1-9.

[8] Carbone, M., Nielsen, M., Sassone, V. 2004. A Calculus for Trust Management, Proceedings of the 2004 Foundations of Software Technology and Theoretical Computer Science (FSTTCS 2004), Chennai, India, 16-18 December 2004 pp.161-173.

[9] Buchegger, S., Le Boudec, J.Y. 2002. Performance analysis of the CONF1DANT protocol: Cooperation of nodes fairness in dynamic ad-hoc networks, Proceedings of the $3^{\text {rd }}$ ACM International Symposium on Mobile Ad Hoc Network and Computing (MobiHOC 2002), Lausanne, Switzerland, 9 11 June 2002 , pp. 226-236.

[10] Golbeck, J., Parsia, B., Hendler, J. A. 2003. Trust Networks on the Semantic Web, Proceedings of the Twelfth International World Wide Web Conference (WWW2003), Budapest, Hungary, 20-24 May 2003.

[11] Xiong, L., Liu, L., "PeerTrust: Supporting Reputation-Based Trust for Peer-to-Peer Electronic Communities", IEEE
Transactions On Knowledge and Data Engineering, Volume 16. Issue 7, USA, July 2004, pp.843-857.

[12] Dewan, P., Dasgupta, P. 2005. Securing P2P Networks Using Peer Reputations: Is there a silver bullet? Proceedings of the 2005 IEEE Consumer Communications and Networking Conference (CCNC 2005), Las Vegas, USA 3-6 January 2005, pp. 30-36

[13] Dragovic, B., Kotsovinos, E., Hand, S, Pietzuch, P. 2003. XenoTrust: Event-based distributed trust management, Proceedings of the $14^{\text {th }}$ Intemational Workshop on Database and Expert Systems Applications (DEXA.03), Prague, Czech Republic, 1-5 September 2003, pp. 410-414

[14] Theodorakopoulos, G., Baras, J.S., "On Trust Models and Trust Evaluation Metrics for Ad Hoc Networks", IEEE Journal on Selected Areas in Communications, Volume 24, 1ssue 2, USA, February 2006, pp.318-328.

[15] Sherwood, R., Lee, S., Bhattacharjee, B, "Cooperative peer groups in NICE", Computer Networks, Volume 50, Issue 4, Elsevier Science, March 2006, pp.523 -544.

[16] Liu, L., Zhang, S., Ryu, K.D., and Dasgupta, P. 2004. RChain: A Self-Maintained Reputation Management System in P2P Networks, Proceedings of the $17^{\text {th }} 1 \mathrm{SCA}$ International Conference on Parallel and Distributed Computing Systems (ISCA 2004), California, USA, 15-17 September 2004

[17] Jurca, R, Faltings, B. 2002. Towards Incentive-Compatible Reputation Management. Trust, Reputation, and Security, Proceedings of the First International Joint Conference on Autonomous Agents and Multi-Agent System (AAMAS 2002), Bologna, Italy, 15-19 July 2002, pp 38-147.

[18] Srivatsa, M., Xiong, L., Liu, L, 2005. TrustGuard: countering vulnerabilities in reputation management for decentralized overlay networks, Proceedings of the $14^{\text {th }}$ International Conference on World Wide Web (WWW 2005), Chiba, Japan, 10-14 May 2005, pp. 422-431

[19] Song, W, Phoha, V.V. 2004. Neural Network-Based Reputation Model in a Distributed System, Ptoceedings of the 2004 IEEE International Conference on E-Commerce Technology (CEC 2004), San Diego, USA, 6-9 July 2004, pp.321-324.

[20] Yu, T., Lin, K.I. 2004. The Design of QoS Broker Algorithms for QoS-Capable Web Services, Proceedings of the 2004 IEEE International Conference on e-Technology, eCommerce, and e-Services (EEE 04), Taipei, Taiwan, 29-31 March 2004, pp. 17-24.

[21] Lin, K.J., Lu, H., Yu, T., Tai, C. 2005. A Reputation and Trust Management Broker Framework for Web Applications, Proceedings of the 2004 IEEE International Conference on e-Technology, e-Commerce, and e-Services (EEE 05), Hong Kong, China , 29 March - I April 2005, pp. 262-269. 